exist, then $0.3 \%$ of infants at private nurseries were anaemic, compared with $3.7 \%$ in Local Authority nurseries, and $15 \%$ of children taken into care.

The relationship of haemoglobin level and hypochromia to iron response was studied.

\section{Reaction to long-term oral anticoagulant therapy. A comparison of prothrombin and partial \&. umboplastin tests}

\section{R. D. EASTH:M (Bristol)}

Over a period of three years 2,178 blood samples were tested from 88 patients, who between them underwent a total of 170 years of long-term oral anticoagulant treatment. The prothrombin ratio and the corresponding activated partial thromboplastin clotting time (PTT) were estimated on each blood sample, the daily dosage of oral anticoagulant being regulated to maintain the PTT between 50 and 70 seconds regardless of the prothrombin ratio. From the results obtained during each year of treatment from each patient the PTT corresponding to a standard prothrombin ratio of 2.0 was calculated. This PTT result corresponding to a reference prothrombin ratio was significantly shorter in patients treated following attacks of venous thrombosis than in patients treated following myocardial infarction or in patients with mitral valve disease treated following embolic attacks. This reference PTT was even shorter in patients in whom a frank attack of acute thrombophlebitis developed during anticoagulant treatment, whatever the original condition necessitating anticoagulant treatment.

These results, taken with the known marked prolongation of the PTT by heparin, suggest that either a 'thromboplasticlike' substance or an anti-heparin substance is released into the circulation during an acute episode of thrombophlebitis, which results in reduction in the PTT unless larger doses of oral anticoagulant are given. It is also suggested that these findings might indicate longer periods of treatment with oral anticoagulants following venous thrombosis.

\section{Book reviews}

Homeostatic Regulators Edited by G. E. W. Wolstenholme and J. Knight (Pp. viii + 329; illustrated.) 75s. London: J. and A. Churchill. 1969.

This book constitutes the edited proceedings of a symposium organized jointly by the Ciba Foundation and the Wellcome Trust early in 1969.

The subjects discussed ranged over a wider field than might have been expected from the title of the symposium. Presentations on cell population kinetics (L. F. Lamerton) and chalones (O. H. Iversen) were to be expected. Less expected were discussions on regulatory systems in cell culture (M. G. P. Stoker), histones (E. W. Johns), nerve growth factors (C. A Vernon and colleagues), cell surface structure and contact control (J. A. Forrester, L. Wolpert, and D. Gingell), and regulatory mechanisms in antibody synthesis (G. Möller). Surprisingly, hor-
Cholestatic jaundice following treatment of chronic granulocytic leukaemia with busul phan

J. C. E. UNDERWOOJ, R. T. SHAHANI, AND E. K. BLACKBURN (Sheffield)

A 25-year-old male with chronic granulocytic leukaemia developed cholestatic jaundice after over six years' treatment? with busulphan, the total dose of this drug being $4.075 \mathrm{~g}$ of which $0.7635 \mathrm{~g}$ was్లు given in the 12 months preceding the onset of jaundice as compared with an annual average intake of $0.6623 \mathrm{~g}$ of the $\overrightarrow{\vec{u}}$ drug during the previous five years. This virtually coincided with the onset of acute relapse. Histological findings at necropsy, while confirming acute relapse of leu kaemia with only minimal hepatic in-0 volvement were, however, also those opo cholestatic jaundice consistent with drug hypersensitivity. We have not found in the literature a similar instance of cho윽 lestatic jaundice probably due to busulphan.- mones are only discussed in passing. Both the definitions of homeostasis given in Stedman's Medical Dictionary (1 The state of equilibrium in the living body with respect to various functions and to the chemical composition of the fluids and tissues, eg, temperature, heart rate, blood pressure, water content, blood sugar, etc. 2 The process through which such body equilibrium is maintained) obviously refer to whole living organisms. The application of the term to regulatory mechanisms at the level of individual organs or tissues and to feedback control mechanisms at the subcellular level, as studied in cultured cells or by purely biochemical methods, seems to deprive it of much of its conceptual value.

Notwithstanding this criticism the book is valuable, because it highlights the poverty of our knowledge of homeostatis (by Stedman's definition) and because it provides an excuse for considering the role of factors such as interferon (N. B. Finter and D. C. Burke) and lysosomes (P. J. Jacques) as regulators. A remarkable feature is the professional quality of the editing which makes the discussions after each paper well worth reading.

FRANCIS J. C. ROE
Die progressiv-dystropischen Myopathien By H. Heyck and G. Laudahn. (Pp. Xv $+\overrightarrow{\bar{T}}$ 436; illustrated. DM118.00.) Berlin, Heidelberg, and New York: SpringerVerlag. 1969.

In the past decade there has been a great increase of interest in the pathophysiology of skeletal muscle, largely as a result of the stimulus provided by variouso organizations devoted to research into muscular dystrophy. One outcome of thiso has been the publication of several first rate monographs upon the myopathies? among which must now be included the comprehensive analysis written by HeyckN and Laudahn.

The first chapter, an historical and clinical review of muscular dystrophy, $\omega$ stems from Heyck's long interest in this subject as chief of the Department of Neurology in the Rudolf-Virchow Hospita in Berlin. He has also written the histo pathological section, with the cooperation of Lüders (pathologist at the Wenckebach Hospital). The ultrastructural changes $\vec{D}$ noted in muscular dystrophy are wel described by Freund-Mölbert. Other尺 chapters are devoted to the 'distal' $\sigma$ 'ocular', and 'menopausal' myopathies, 